\title{
Electroplated Ni-CNT Nanocomposite for Micromechanical Resonator Applications
}

\author{
Yi-Chia Lee, Ming-Huang Li, Y. T. Cheng, Senior Member, IEEE, Wensyang Hsu, and Sheng-Shian Li
}

\begin{abstract}
In this letter, electroplated Ni-CNT nanocomposite is synthesized and utilized as a structural material for micromechanical resonator fabrication. Pretreated by sulfate sodium dodecyl, carbon nanotubes (CNTs) disperse well in electrolyte and can be incorporated in an electroplated $\mathrm{Ni}$ film. Clamped-clamped beam resonators fabricated using the nanocomposite show $\sim 27 \%$ resonant frequency enhancement (from 498.75 to $634.72 \mathrm{kHz}$ ). Meanwhile, the $Q$ factors of 781 and 760 for $\mathrm{Ni}$ and Ni-CNT nanocomposite resonators, which are comparable with the prior art, indicate that the anchor loss could dominate the $Q$ performance of the beam resonators.
\end{abstract}

Index Terms-Electroplated Ni-CNT nanocomposite, microelectromechanical systems (MEMS), quality factor, resonant frequency, resonator.

\section{INTRODUCTION}

M ICROMECHANICAL resonators have begun replacing their quartz counterparts in timing and clock control for system-on-chip applications because they exhibit many technical advantages, particularly in terms of size, cost, and system integration [1], [2]. Since the microelectromechanical systems (MEMS)-last scheme is fully CMOS compatible, it has been proposed to be the best economic strategy for integrating MEMS resonators and transistors onto a single chip [3], [4]. However, as CMOS technology advances, the low- $k$ dielectric materials used in the back end of the line may not survive if the post-CMOS processing temperature is over $400{ }^{\circ} \mathrm{C}$ [5]. Since the processing temperature in conventional high-quality CVD poly-SiGe [3] or poly-Si processes [4] for micromechanical resonator fabrication is close to or even higher than the ceiling temperature, it is required to develop new structural materials that can have low processing temperatures as well as maintain the performance of micromechanical resonators, such as operational frequency and quality factor $(Q)$. Electroplated Ni has been recognized as a candidate material for micromechanical

Manuscript received December 9, 2011; revised February 18, 2012, February 24, 2012, and February 27, 2012; accepted February 29, 2012. Date of publication April 16, 2012; date of current version May 18, 2012. This work was supported in part by the National Science Council (NSC) under Grants 100-2220-E-009-007 and NSC 97-2221-E-009-020-MY3 and in part by the Ministry of Education in Taiwan under the ATU Program. The review of this letter was arranged by Editor E. A. Gutiérrez-D.

Y.-C. Lee and W. Hsu are with the Department of Mechanical Engineering, National Chiao Tung University, Hsinchu 300, Taiwan (e-mail: hook.me94g@nctu.edu.tw; whsu@mail.nctu.edu.tw).

M.-H. Li and S.-S. Li are with the Institute of NanoEngineering and MicroSystems, National Tsing Hua University, Hsinchu 30013, Taiwan (e-mail: s100035807@m100.nthu.edu.tw; ssli@mx.nthu.edu.tw).

Y.-T. Cheng is with the Microsystems Integration Laboratory, Department of Electronics Engineering, National Chiao Tung University, Hsinchu 300, Taiwan (e-mail: ytcheng@mail.nctu.edu.tw).

Color versions of one or more of the figures in this letter are available online at http://ieeexplore.iee.org.

Digital Object Identifier 10.1109/LED.2012.2190131 resonator fabrication owing to its low processing temperatures and good mechanical properties [6], [7]. The processing temperature of electroplated Ni required for the MEMS-last integration can be as low as $50{ }^{\circ} \mathrm{C}$ [6], much lower than $450^{\circ} \mathrm{C}$ ceiling for postprocessing [3]. Furthermore, the Ni disktype micromechanical resonator can maintain its $Q$ factor in the thousands [6] or even tens of thousands for a more aggressive resonator design [7].

Carbon nanotubes (CNTs) have many extraordinary characteristics, including high tensile strength, large elastic modulus, excellent thermal and electrical conductivities, and high aspect ratio in structures, enabling potential applications in synthesizing new composite materials. Several efforts have been made not only to synthesize but also to characterize the composites using the CNTs as the key ingredient [8]-[15]. Among these studies, the phenomenon of mechanical strengthening has been found by incorporating CNTs into metal matrices [10]-[15]. CNTs can be incorporated into a Ni matrix via electroplating or electroless deposition to improve the properties of $\mathrm{Ni}$ such as wear resistance [10], thermal conductivity [11], tensile strength [12], hardness [13], and elastic modulus [14], [15]. Unfortunately, for the composite synthesis, CNTs are often in a form of entangled agglomerates.

In order to fully adopt the unique properties of CNTs, many pretreatments have been developed for nanocomposite deposition. Among these pretreatments, noncovalent functionalization treated by surfactant has been the most prevalent one [11]-[13], [16]. Therefore, sulfate sodium dodecyl (SDS), one of the most popular surfactants for CNT dispersion in a water solution [12], [13], [16], is employed in this work to pretreat CNTs in order to keep good particle-dispersion characteristics in the electrolyte. By electroplating, Ni-CNT clamped-clamped-nanocomposite beams (CC-nanocomposite beams) are fabricated to demonstrate the synthesis process for MEMS fabrication, and the resultant performance improvement of the CC-beam resonator from CNT incorporation is also investigated.

\section{Device FABRication}

Due to a large stiffness-to-mass ratio, CC-beam mechanical resonators have been intensively investigated for very high frequency range applications [17], [18]. In this letter,we utilized the CC beam as the device structure for the characterization of the Ni-CNT nanocomposite resonators by measuring their first/flexural mode. Fig. 1 shows the cross sections of the $\mathrm{CC}$-beam resonator fabricated with electroplated $\mathrm{Ni}$ or $\mathrm{Ni}-$ CNT nanocomposite before and after structural release. The fabrication started with the growth of thermal oxide $(500 \mathrm{~nm})$ on a $\langle 100\rangle$ silicon wafer as an electrical isolation layer, followed by sputtering $\mathrm{Ti} / \mathrm{Ni}(20 / 150 \mathrm{~nm})$ and $\mathrm{Cu}(500 \mathrm{~nm})$ as the device 


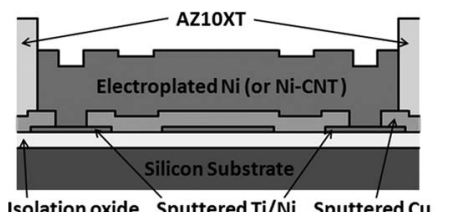

as-fabricated

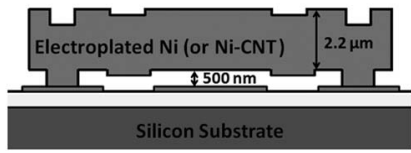

as-released

Fig. 1. Cross-sectional schemes of the low-temperature electroplated $\mathrm{Ni}$ (or Ni-CNT) CC-beam resonator before and after structural release.

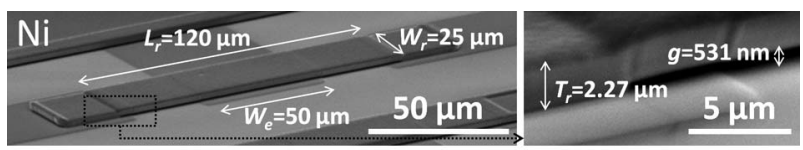

(a)

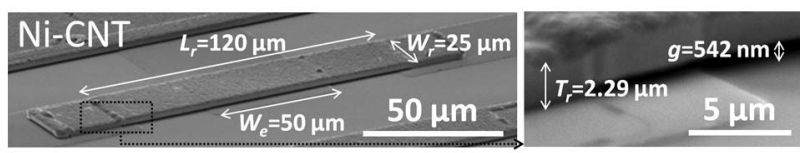

(b)

Fig. 2. SEM images of (a) Ni and (b) Ni-CNT CC-beam resonators.

electrode and sacrificial layer that were also utilized as the seed layer for electroplating the anchors and suspended parts of the resonator. The electrode and sacrificial layers were both patterned by liftoff processes. Before electroplating, a layer of thick photoresist, AZ10XT $(>5 \mu \mathrm{m})$, was spun and patterned as the mold where the Ni-based film was electroplated to form the resonator beam. Finally, the resonator fabrication was finished after the removal of the $\mathrm{PR}$ mold and $\mathrm{Cu}$ sacrificial layer by acetone and the mixture of $\mathrm{NH}_{4} \mathrm{OH}$ and $\mathrm{H}_{2} \mathrm{O}_{2}(4: 1)$, respectively. Due to good etching selectivity of the mixture of $\mathrm{NH}_{4} \mathrm{OH}$ and $\mathrm{H}_{2} \mathrm{O}_{2}$, the $\mathrm{Cu}$ sacrificial layer was removed completely without damaging the Ni-based structure. The beam length $\left(L_{r}\right)$, width $\left(W_{r}\right)$, and thickness $\left(T_{r}\right)$ of the resonator are 120,25 , and $2.2 \mu \mathrm{m}$, respectively. In addition, the thickness of the sacrificial $\mathrm{Cu}$ layer $(g)$ and the width of the electrode $\left(W_{e}\right)$ are $500 \mathrm{~nm}$ and $50 \mu \mathrm{m}$, respectively.

The CNTs used in this work are a commercial product (Seedchem Pty., Ltd.). The tubes with $95 \%$ purity are prepared by a chemical vapor deposition method. The inner and outer diameters of the CNTs are on the average of 5-10 and 10-20 nm, respectively. The electrolyte was composed of nickel sulfamate of $400 \mathrm{~g} / \mathrm{L}$, boric acid of $40 \mathrm{~g} / \mathrm{L}$, nickel chloride of $3 \mathrm{~g} / \mathrm{L}$, and wetting agent of $5 \mathrm{ml} / \mathrm{L}$. After putting the CNTs $(1 \mathrm{~g} / \mathrm{L})$ and the SDS $(0.6 \mathrm{~g} / \mathrm{L} \mathrm{[16])} \mathrm{into} \mathrm{the} \mathrm{electrolyte,} \mathrm{ultrasonication} \mathrm{is}$ performed for $120 \mathrm{~min}$ to ensure that the CNTs can be separated from each other and fully dispersed in the electrolyte. From the measurement by dynamic-light-scattering spectroscopy, the length of the dispersed CNTs in the electrolyte is in the range of $0.5-10 \mu \mathrm{m}$. The plating process was done at the temperature of $35{ }^{\circ} \mathrm{C}$ with a current density of $1.5 \mathrm{~mA} / \mathrm{cm}^{2}$.

\section{RESULTS AND DiscUSSION}

Fig. 2 shows the SEM images of Ni [see Fig. 2(a)] and NiCNT [see Fig. 2(b)] CC beams. The measured thickness of the structure and sacrificial layers are $2.27 \mu \mathrm{m}$ and $531 \mathrm{~nm}$ for the Ni CC beams and $2.29 \mu \mathrm{m}$ and $542 \mathrm{~nm}$ for the Ni-CNT one, respectively. Each electroplating process was controlled at $60 \mathrm{~min}$, so the deposition rates of electroplated $\mathrm{Ni}$ and $\mathrm{Ni}$ -

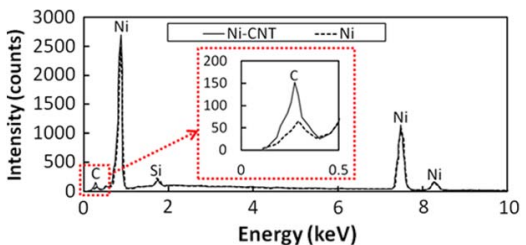

(a)

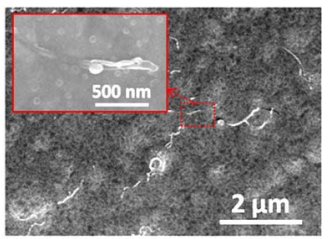

(b)
Fig. 3. (a) EDS analysis of the Ni and the Ni-CNT film. (b) High-resolution SEM images of Ni-CNT film showing that CNT has one end embedded inside the matrix as a root and the other end pointing outward as a tip.

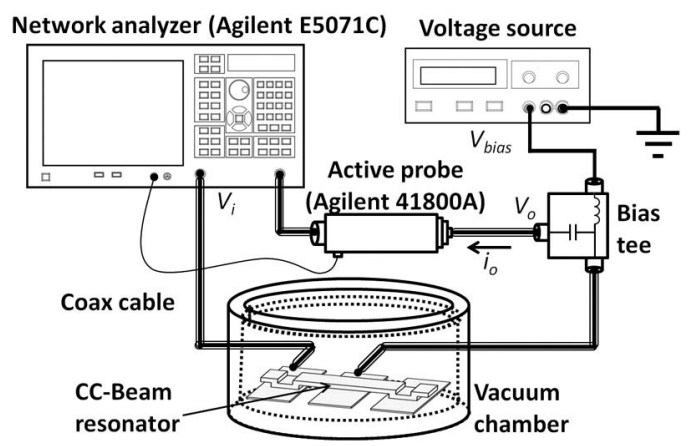

(a)

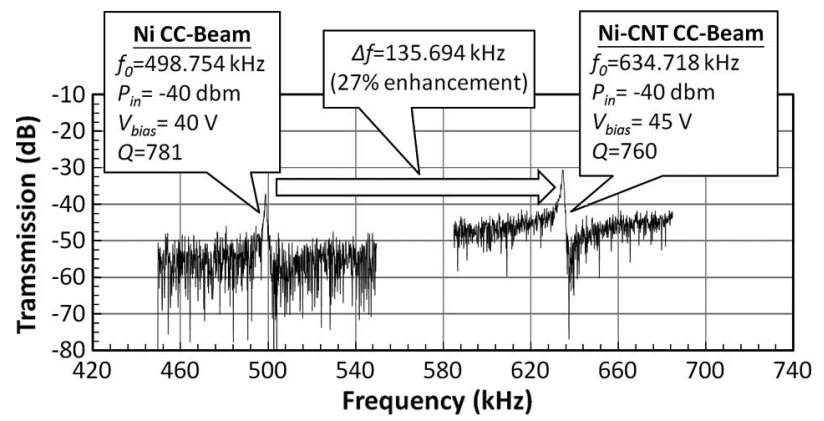

(b)

Fig. 4. (a) Frequency characteristic measurement scheme of the CC-beam resonator. (b) Comparison of the frequency characteristics of $\mathrm{Ni}$ and $\mathrm{Ni}-\mathrm{CNT}$ CC-beam resonators at 0.2 mtorr.

CNT are derived as 37.8 and $38.2 \mathrm{~nm} / \mathrm{min}$, respectively. After completely removing the $\mathrm{Cu}$ sacrificial layer, no structural deformation is found, and the resonator beam can be freely suspended over the electrode.

Fig. 3(a) shows the analysis of energy dispersive spectroscopy (EDS), which detects the elements in the film surface. The enlarged figure in Fig. 3(a) shows that the carbon peak of Ni-CNT is more than two times higher than that of Ni. The increase of carbon intensity can be attributed to the incorporation of CNTs. Fig. 3(b) shows the SEM images of the as-fabricated Ni-CNT CC beam where CNTs are well distributed and partly engulfed in the Ni matrix. Every nanotube is separated from each other, and the agglomeration of CNTs is not observed. The resonant frequency of the beam structure was premeasured by a white light interferometer (LDV, Ono Sokki Company, Ltd.) for Young's modulus/density $(E / \rho)$ characterization. The LDV-measured resonant frequencies are 619.38 and $760.88 \mathrm{kHz}$ for the $\mathrm{Ni}$ and $\mathrm{Ni}-\mathrm{CNT}$ nanocomposite beams, respectively. From the modal simulation of FEA software (Ansys) regarding the frequency responses, the $E / \rho$ ratios are $1.51 \times 10^{7}$ and $2.24 \times 10^{7} \mathrm{~Pa} \cdot \mathrm{m}^{3} / \mathrm{kg}$ for the $\mathrm{Ni}$ and Ni-CNT 
nanocomposite, respectively. About $48 \%$ enhancement of the $E / \rho$ ratio has been realized.

Fig. 4(a) presents the CC-beam resonator with a typical driving and sensing setup. The resonator consists of a movable flexural beam clamped at both ends and a driving electrode underneath the beam. A dc bias voltage $\left(V_{\text {bias }}\right)$ is applied to the resonator, while an ac excitation voltage $\left(V_{i}\right)$ is applied to the electrode. When the frequency of $V_{i}$ matches the resonant frequency of the resonant beam, the resonator begins to vibrate and forms a time-varying capacitor. This time-varying capacitor biased with the DC voltage could generate an output current $\left(i_{o}\right)$. The frequency characteristic was collected using a network analyzer (Agilent Technologies Inc., E5071C) with an active probe (Agilent Technologies Inc., 41800A) for matching the impedance between the input port of the network analyzer and the output of the resonator. The CC-beam resonator is placed into a vacuum chamber, and the measurement is performed at a pressure under 0.5 mtorr.

Fig. 4(b) presents the frequency characteristics of $\mathrm{Ni}$ and $\mathrm{Ni}$-CNT CC-beam resonators at 0.2 mtorr. It shows resonant frequencies $\left(f_{0}\right)$ of 498.75 and $634.72 \mathrm{kHz}$ for the $\mathrm{Ni}$ and $\mathrm{Ni}$ CNT CC-beam resonators, respectively, designed with the same dimensions. About 27\% frequency enhancement can be realized and attributed to the incorporation of CNTs. In addition, it can be found that the electrical sensing results are different from that of the LDV measurement. This frequency reduction is caused by the introduction of electrical stiffness $\left(k_{e}\right)$ related to the interaction of the electric field between the resonator and drive electrode, and hence, the effect would lower the effective spring stiffness as follows [19]:

$$
f_{0}=\frac{1}{2 \pi} \sqrt{\frac{k_{r}-k_{e}}{m_{r}}}=\frac{1}{2 \pi} \sqrt{\frac{k_{r}-\left(V_{\mathrm{bias}}^{2} \varepsilon_{0} W_{e} W_{r} / g^{3}\right)}{m_{r}}}
$$

where $k_{r}$ and $m_{r}$ are the mechanical spring constant and mass of the resonator, respectively. From (1), the electrical spring constants of the $\mathrm{Ni}$ and $\mathrm{Ni}-\mathrm{CNT}$ resonators are calculated as 118.27 and $140.76 \mathrm{~N} / \mathrm{m}$, so the calculated resonant frequencies of the resonators applied with $V_{\text {bias }}$ are 503.65 and $639.46 \mathrm{kHz}$, which are close to the aforementioned measurements.

The measured $Q$ values of the $\mathrm{Ni}$ and Ni-CNT CC-beam resonators are 781 and 760 , respectively, indicating that the CNT incorporation did not cause the $Q$ degradation. The $Q$ performance of the Ni CC-beam resonator, i.e., 781, is as good as the prior art [7]. The anchor loss is known to dominate the $Q$ performance of the CC-beam resonators [20]. In comparison with the $Q$ performance of the polysilicon CC-beam resonators [21], the lower $Q$ performance of the Ni-based resonators may be a result of poor adhesion of the beam anchors to the substrate underneath, leading to more energy dissipation during vibration [7]. Further performance improvement, particularly in $Q$, can be realized by employing different resonator designs like free-free beam or wineglass disk resonators [6], [7], [22].

\section{CONCLUSION}

In summary, an electroplating process has been successfully demonstrated for Ni-CNT nanocomposite CC-beam resonator fabrication. The entire process takes place at temperatures lower than $90{ }^{\circ} \mathrm{C}$ such that this process is compatible with the CMOS process. By employing the SDS treatment on the surface of CNTs for good dispersion characteristics, the incorporation of CNTs into the Ni matrix results in Young's modulus-todensity ratio enhancements of $\sim 48 \%$ and resonant frequency increases of $\sim 27 \%$ while maintaining $Q$.

\section{REFERENCES}

[1] W. T. Hsu, "Reliability of silicon resonator oscillator," in Proc. IEEE Int. Freq. Control Symp. Expo., Miami, FL, Jun. 2006, pp. 389-392.

[2] M. Lutz, A. Partridge, P. Gupta, N. Buchan, E. Klaassen, J. McDonald, and K. Petersen, "MEMS oscillators for high volume commercial applications," in Proc. TRANSDUCERS, Lyon, France, Jun. 2007, pp. 49-52.

[3] A. E. Franke, J. M. Heck, K. Tsu-Jae, and R. T. Howe, "Polycrystalline silicon-germanium films for integrated microsystems," J. Microelectromech. Syst., vol. 12, no. 2, pp. 160-171, Apr. 2003.

[4] C. T. C. Nguyen and R. T. Howe, "An integrated CMOS micromechanical resonator high-Q oscillator," IEEE J. Solid-State Circuits, vol. 34, no. 4, pp. 440-455, Apr. 1999.

[5] G. Maier, "The search for low-k and ultra-low-k dielectrics: How far can you get with polymers? Part 1: Background," IEEE Electr. Insul. Mag., vol. 20, no. 2, pp. 6-17, Dec. 2004.

[6] W. L. Huang, Z. Ren, Y. W. Lin, H.-Y. Chen, J. Lahann, and C. T.-C. Nguyen, "Fully monolithic CMOS nickel micromechanical resonator oscillator," in Proc. 21th IEEE Int. Conf. Micro Electro Mech. Syst., Tucson, AZ, Jan. 13-17, 2008, pp. 10-13.

[7] W. L. Huang, Z. Ren, and C. T.-C. Nguyen, "Nickel vibrating micromechanical disk resonator with solid dielectric capacitive-transducer gap," in Proc. IEEE Int. Freq. Control Symp., Miami, FL, Jun. 5-7, 2006, pp. 839-847.

[8] O. Laurie and H. D. Wagner, "Evaluation of Young's modulus of carbon nanotubes by micro-Raman spectroscopy," J. Mater. Res., vol. 13, no. 9 , pp. 2418-2422, Sep. 1998.

[9] H. Cai, F. Yan, and Q. Xe, "Investigation of tribological properties of polyimide/carbon nanotube nanocomposites," Mater. Sci. Eng., A, vol. 364, no. 1/2, pp. 94-100, Jan. 2004.

[10] X. H. Chen, C. S. Chen, H. N. Xiao, H. B. Liao, L. P. Zhou, S. L. Li, and G. Zhang, "Dry friction and wear characteristics of nickel/carbon nanotube electroless composite deposits," Tribol. Int., vol. 39, no. 1, pp. 22-28, 2006.

[11] S. Arai, M. Endo, T. Sato, and A. Koide, "Fabrication of nickelmultiwalled carbon nanotube composite films with excellent thermal conductivity by an electrodeposition technique," Electrochem. Solid-State Lett., vol. 9, no. 8, pp. C131-C133, 2006.

[12] Y. Sun, J. Sun, M. Liu, and Q. Chen, "Mechanical strength of carbon nanotube-nickel nanocomposites," Nanotechnology, vol. 18, no. 50, pp. 505 704-1-505 704-6, 2007.

[13] Y. S. Jeon, J. Y. Byun, and T. S. Oh, "Electrodeposition and mechanical properties of Ni-carbon nanotube nanocomposite coatings," J. Phys. Chem. Solids, vol. 69, no. 5/6, pp. 1391-1394, May 2008.

[14] G. R. Shen, Y. T. Cheng, and L. N. Tsai, "Synthesis and characterization of Ni-P-CNT's nanocomposite film for MEMS applications," IEEE Trans. Nanotechnol., vol. 4, no. 5, pp. 539-547, Sep. 2005.

[15] L. N. Tsai, Y. T. Cheng, and W. Hsu, "Ni-carbon nanotubes nanocomposite for robust microelectromechanical systems fabrication," J. Vac. Sci. Technol. B, Microelectron. Nanometer Struct., vol. 24, no. 1, pp. 205-210, Jan. 2006.

[16] C. Guo, X. Zhao, J. Zhao, and J. Xiong, "Effects of surfactants on electrodeposition of nickel-carbon nanotubes composite coatings," Surf. Coatings Technol., vol. 202, no. 14, pp. 3385-3390, Apr. 2008.

[17] C. T. C. Nguyen, "Frequency-selective MEMS for miniaturized lowpower communication devices," IEEE Trans. Microw. Theory Tech., vol. 47, no. 8, pp. 1486-1503, Aug. 1999.

[18] V. Kaajakari, J.iihamäki, A. Oja, S. Pietikäinen, V. Kokkala, and H. Kuisma, "Stability of wafer level vacuum encapsulated single-crystal silicon resonators," in Proc. TRANSDUCERS, Seoul, Korea, Jun. 2005, pp. 916-919.

[19] W. T. Hsu, "Vibrating RF MEMS for timing and frequency references," in Proc. IEEE Int. Microw. Theory Tech. Symp., San Francisco, CA, Jun. 2006, pp. 672-675.

[20] Y. H. Park and K. C. Park, "High-fidelity modeling of MEMS resonators-Part I: Anchor loss mechanisms through substrate," J. Microelectromech. Syst., vol. 13, no. 2, pp. 238-247, Apr. 2004.

[21] F. D. Bannon, J. R. Clark, and C. T. C. Nguyen, "High-Q HF microelectromechanical filters," IEEE J. Solid-State Circuits, vol. 35, no. 4, pp. 512-526, Apr. 2000.

[22] K. Wang and C. T. C. Nguyen, "VHF free-free beam high-Q micromechanical resonators," J. Microelectromech. Syst., vol. 9, no. 3, pp. 374360 , Sep. 2000 